\title{
P 210 SURVEY OF PATIENT SATISFACTION WITH MEDICAL CARE ON A HOSPICE INPATIENT UNIT
}

Helen Lucraft, Paul McNamara. St Oswald's Hospice, Newcastle upon Tyne, UK

10.1136/bmjspcare-2014-000654.251

Background Unlike our previous inpatient surveys, we focussed solely on the medical input. Our medical Clinical improvement volunteer, an experienced clinician, carried out this project.

Aims To survey 40 inpatients on their experience of our medical input.

From Aug '12-Feb'13, forty inpatients completed the questionnaire. 5 of the 10 questions (asterisked*) were benchmarked against national comparators. This benchmarking was undertaken by the Centre for Health Service Research, University of Kent, in 2004/5 and 2008/9.

Q1. How soon after arrival did the doctor see you? Q2. Was the doctor polite and courteous?" Q3. Did the doctor understand your priorities and concerns?* Q4. Were you able to ask the doctors questions when you wanted?* Q5. Did the doctors explain what they thought was happening?* Q6. Are the doctors available to see your family? Q7. How would you rate your medical care?* Q8. Any aspect of your medical care you are unhappy with? Q9. If you could change one thing in your medical care? Q10. Any comments or suggestions for the doctors?

Results In the five questions which were nationally benchmarked, (asterisked*), our results compared favourably, exceeding them in each case. Qs $1 \& 6$ also scored highly.

The final three questions, asking patients to critique their medical input, only provided limited suggestions. This made us wonder if it is difficult to make critical comments; however this was done with a volunteer, not a staff member.

Patient concerns expressed were calls for consistent daily medical review and patient anxieties around discharge home.

Conclusions We were heartened that inpatients felt overwhelmingly positive with their medical care. We have responded to the comment about consistent review and we aim to repeat the survey in eighteen months. 